\title{
Efeito do tamanho dos sedimentos sobre a área atingida por fluxos de detritos: Estudo de caso da bacia do arroio Böni, Serra Gaúcha
}

\author{
Sediment size effect on debris flow runout area: Case study of Böni watershed, Serra Gaucha
}

\author{
Maurício Andrades Paixão e Masato Kobiyama
}

Instituto de Pesquisas Hidráulicas, Universidade federal do Rio Grande do Sul, RS - Brasil

mauricio.paixao@ufrgs.br; masato.kobiyama@ufrgs.br

\section{Resumo}

O presente trabalho teve por objetivo avaliar o efeito do tamanho dos sedimentos sobre a área atingida por fluxos de detritos por meio do uso de Kanako-2D, utilizando como estudo de caso a bacia do arroio Böni na Serra Gaúcha. Tal bacia hidrográfica apresenta vasto histórico de recorrência de desastres naturais, em especial os ocorridos nos anos 1982 e 2000. Foram utilizados os diâmetros de 0,15, 0,30, 0,50, 1,0, 1,3, 2,0 e 3,0 m nas simulações e avaliadas as áreas de erosão e deposição, bem como a área total atingida. O tamanho dos sedimentos influenciou fortemente as áreas de erosão, deposição e a área total atingida. Sedimentos menores apresentaram maior mobilidade, percorrendo maiores distâncias e atingindo maiores áreas. Os resultados obtidos confirmam que a utilização de modelagem computacional na modelagem de fluxos de detritos pode ser importante ferramenta na predição de áreas susceptíveis a estes fenômenos.

Palavras-chave:Fluxo de detritos; Tamanho dos sedimentos; Kanako-2D

\section{Abstract}

The present paper aimed to evaluate the size sediment effects on runout area damaged by debris flow by using Kanako$2 D$ in Böni Watershed, Serra Gaucha. This watershed presents a vast history of natural disasters, highlighting the events occurred in 1982 and 2000. The sediment sizes considered in the simulations and evaluated in terms of erosion, deposition and reached area were 0.15, 0.30, 0.50, 1.0, 1.3, 2.0 and $3.0 \mathrm{~m}$. The sediment size strongly influenced on the erosion, deposition and total area reached by debris flow. Fine-grained sediments presented higher mobility, traveling greater distances and reaching larger areas than coarse-grained sediments. The obtained results confirm that Kanako-2D can be an important tool to predict susceptible areas to debris flow.

Keywords: Debris flow; Sediment size; Kanako-2D 


\section{Introdução}

Fluxos de detritos são fenômenos naturais altamente destrutivos, compostos pela mistura de água, ar e sedimentos, e governados pela gravidade (TAKAHASHI, 2014), sendo um dos tipos de movimento de massas mais perigosos devido à velocidade do fluxo e às longas distâncias que podem percorrer (JAKOB e HUNGR, 2005). Conforme o IRDR (2014), tais fenômenos são um tipo de movimento de massa em que chuvas intensas causam o descenso de uma grande quantidade de detritos em encostas, tais como-, vegetação, rochas e lama. No Brasil também são conhecidos como corridas de massa, por exemplo, em textos do Ministério da Integração Nacional (2012).

Kobiyama et al. (2015) comentaram que fluxos de detritos têm causado diversos danos à sociedade e ao meio ambiente. Podem-se citar os grandes eventos que recentemente ocorreram em Santa Catarina, em 2008 (FRANK e SEVEGNANI, 2009), e na região serrana do Rio de Janeiro, em 2011 (AVELAR et al., 2011; COELHO NETTO et al., 2011). Muitas tragédias poderiam ser evitadas caso a sociedade tivesse acesso a estudos sobre desastres relacionados a fluxo de detritos e reconhecesse áreas susceptíveis a estes desastres.

Uma das medidas para redução deste tipo de desastre baseia-se no mapeamento de áreas propensas aos fluxos de detritos, o que faz necessário aumentar o conhecimento acerca dos processos físicos envolvidos no fenômeno para viabilizar a aplicação de modelos que descrevem seu comportamento (REIS et al., 2014). Assim, surge a importância e necessidade de utilizar a modelagem computacional com base física. Embora haja diversos modelos computacionais no mundo, o modelo Kanako-2D, desenvolvido por Nakatani et al. (2008), é um dos mais utilizados atualmente. O modelo é gratuito e possui interface amigável para quem não domina linguagens de programação, o que pode ajudar na popularização do modelo.

Um dos parâmetros mais utilizados para fins de calibração no mapeamento de áreas susceptíveis a fluxos de detritos no modelo Kanako-2D é o diâmetro dos sedimentos. Conforme Uchida et al. (2013), é necessário obter melhor definição empírica e teórica deste parâmetro e o modelo Kanako-2D é altamente sensível a ele. Portanto, pode-se dizer que o tamanho dos sedimentos influencia fortemente no alcance e na área atingida pelo fluxo de detritos.

Assim sendo, o objetivo do presente trabalho foi avaliar o efeito do tamanho dos sedimentos sobre a área atingida por fluxos de detritos utilizando o Kanako-2D. A bacia hidrográfica do arroio Böni foi escolhida como estudo de caso. Esta bacia está localizada na região serrana do estado do Rio Grande do Sul, em uma região com vasto histórico de ocorrência de fluxos de detritos.

\section{Materiais e métodos}

\section{1 Área de Estudo}

A área de estudo é a bacia hidrográfica do arroio Böni $\left(2,27 \mathrm{~km}^{2}\right.$ ) (Figura 1), compreendida entre os municípios de São Vendelino, Farroupilha e Alto Feliz, no estado do Rio Grande do Sul. Essa bacia está inserida na bacia do rio Caí, a qual é, segundo Comitê Caí (2008), uma das mais importantes economicamente para o estado. A região está localizada entre escarpas e o planalto da Serra Geral, apresentando altas declividades, em ambiente tipicamente montanhoso (GODOY et al., 2015). Segundo Flores et al. (2007), os solos da região pertencem à classe dos Neossolos (RLe 2). Segundo Vieiro e Silva (2010), as unidades litoestratigráficas da região pertencem à Formação Serra Geral, incluindo a Fácies Caxias e Fácies Gramado. A bacia do arroio Böni apresente altimetria entre 379 e $673 \mathrm{~m}$. 


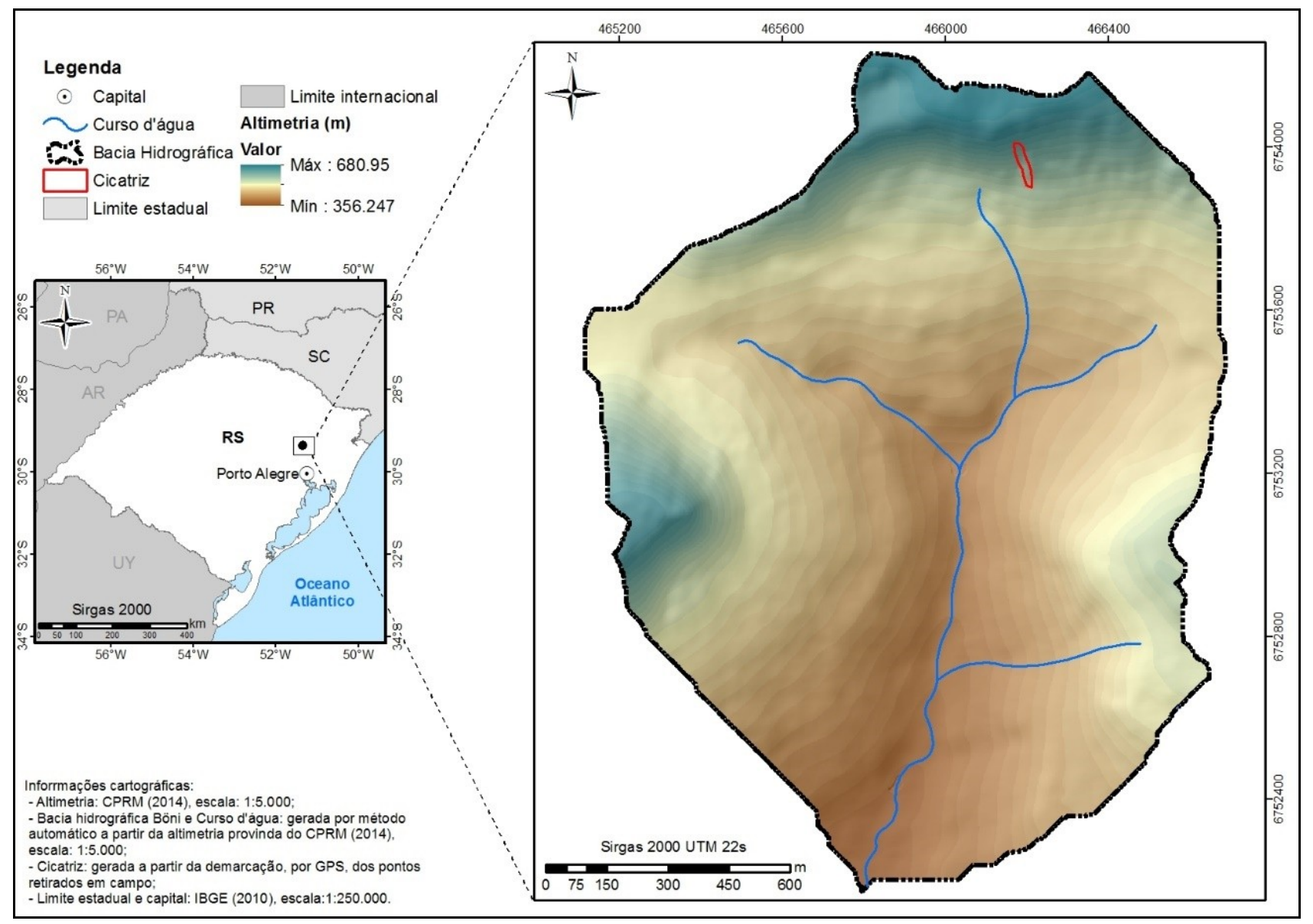

Figura 1 - Bacia hidrográfica do arroio Böni

A região apresenta um vasto histórico de ocorrência de desastres hidrológicos na região (GODOY et al., 2015; MICHEL, 2015), sendo que grandes eventos foram registrados nos anos de 1982 e 2000. Michel (2015) comentou que os movimentos de massa que ocorrem na região são caracterizados por serem escorregamentos translacionais rasos que, ao convergirem para o canal se transformaram em fluxos de detritos.

\subsection{Dados Utilizados}

O modelo digital de elevação (MDE) da bacia do arroio Böni foi obtido junto ao Serviço Geológico do Brasil (CPRM), com resolução de 2,5 x 2,5 m. A identificação das cicatrizes foi realizada a partir de CPRM (2014). $\mathrm{O}$ volume do fluxo de detritos foi calculado a partir do produto entre a área da cicatriz e a profundidade do solo, estimada em $2 \mathrm{~m}$ por Michel (2015). Portanto, o volume do fluxo de detritos $\left(V_{F D}\right)$ utilizado foi de $7500 \mathrm{~m}^{3}$.

\subsection{Aplicação do Kanako-2D}

Os fluxos de detritos apresentam zonas de iniciação, de transporte e de deposição. O modelo Kanako-2D realiza as simulações a partir da zona de transporte, ou seja, a partir de sua entrada no canal de propagação do fluxo. 
$\mathrm{Na}$ zona de transporte a simulação ocorre em $1 \mathrm{D}$, enquanto na zona de deposição a simulação ocorre em $2 \mathrm{D}$ (NAKATANI et al., 2008).

O modelo Kanako-2D utiliza equações de continuidade, momento, deformação do leito do canal, erosão/deposição e tensões de cisalhamento no leito, isto é:

- Equação da continuidade para o volume total:

$$
\frac{\partial h}{\partial t}+\frac{\partial u h}{\partial x}+\frac{\partial v h}{\partial y}=i
$$

- Equação da continuidade para determinação do fluxo na k-ésima posição da partícula i

$$
\frac{\partial C_{k} h}{\partial t}+\frac{\partial C_{k} h u}{\partial x}+\frac{\partial C_{k} h v}{\partial y}=i_{k} \cdot C^{*}
$$

- Equação de conservação de momento nas direções x e y

$$
\frac{\partial(u, v)}{\partial t}+x \frac{\partial(u, v)}{\partial x}+v \frac{\partial(u, v)}{\partial y}=g \cdot \operatorname{sen} \theta_{(x, y)}-\frac{\tau(x, y)}{\rho f h f}
$$

- Equação que avalia as deformações no leito

$$
\frac{\partial z}{\partial t}+i=0
$$

em que $h_{f}$ é a altura do fluxo; $u$ é a velocidade na direção $x$; $v$ é a velocidade na direção $y$; $C_{j}$ é a concentração de sedimentos por volume do fluxo na célula $k$; $z$ é a elevação do leito; $i$ é a taxa de deposição/erosão; $\rho_{f}$ é a massa específica do fluido intersticial; $\theta_{x}$ e $\theta_{y}$ são as declividades nas direções $x$ e $y$; $C^{*}$ é a concentração de sedimentos no leito móvel; $\tau_{x}$ e $\tau_{y}$ são as tensões de cisalhamento no leito nas direções $x$ e $y$.

Devido ao modelo iniciar a simulação a partir da entrada do fluxo no canal, foi necessário estabelecer o hidrograma de sedimentos a ser propagado. Conhecendo-se o volume $\left(7500 \mathrm{~m}^{3}\right)$, utilizou-se o método de Whipple (1992) para determinar a vazão de pico, em que o tempo de ascensão é menor que o tempo de recessão. Michel et al. (2015) adotou tempo de recessão igual ao dobro do tempo de ascensão obtendo resultados satisfatórios. A vazão de pico $(Q p)$ do hidrograma foi determinada a partir da equação proposta por Rickenmann (1999):

$$
Q p=0,1 \cdot V_{F D} 0,833
$$

A fim de avaliar o efeito do tamanho dos detritos na propagação do fluxo, o presente trabalho alterou o tamanho dos sedimentos entre 0,15 e 3,00 m no modelo. Foram avaliados o alcance, a área atingida que é caracterizada com o conjunto de áreas de erosão e sedimentação do fluxo. A Tabela 1 apresenta os valores dos parâmetros de entrada do Kanako-2D utilizados no presente trabalho. 
Tabela 1 - parâmetros utilizados nas simulações

\begin{tabular}{l|c}
\hline Parâmetro & Valores \\
\hline Intervalo do tempo de cálculo $(\mathrm{s})$ & 0,01 \\
\hline Diâmetro dos sedimentos $(\mathrm{m})$ & 0,$15 ; 0,30 ; 0,50 ; 1,0 ; 1,3 ; 2,0 ; 3,0$ \\
\hline Massa específica do leito $\left(\mathrm{kg} / \mathrm{m}^{3}\right)$ & 2650 \\
\hline Massa específica da fase fluida $\left(\mathrm{kg} / \mathrm{m}^{3}\right)$ & 1250 \\
\hline Concentração do leito móvel $(\mathrm{g} / \mathrm{L})$ & 0,6 \\
\hline Coeficiente de erosão & 0,0007 \\
\hline Coeficiente de sedimentação & 0,05 \\
\hline Coeficiente de rugosidade de Manning & 0,03 \\
\hline
\end{tabular}

O funcionamento simplificado do Kanako-2D pode ser visualizado na Figura 2.

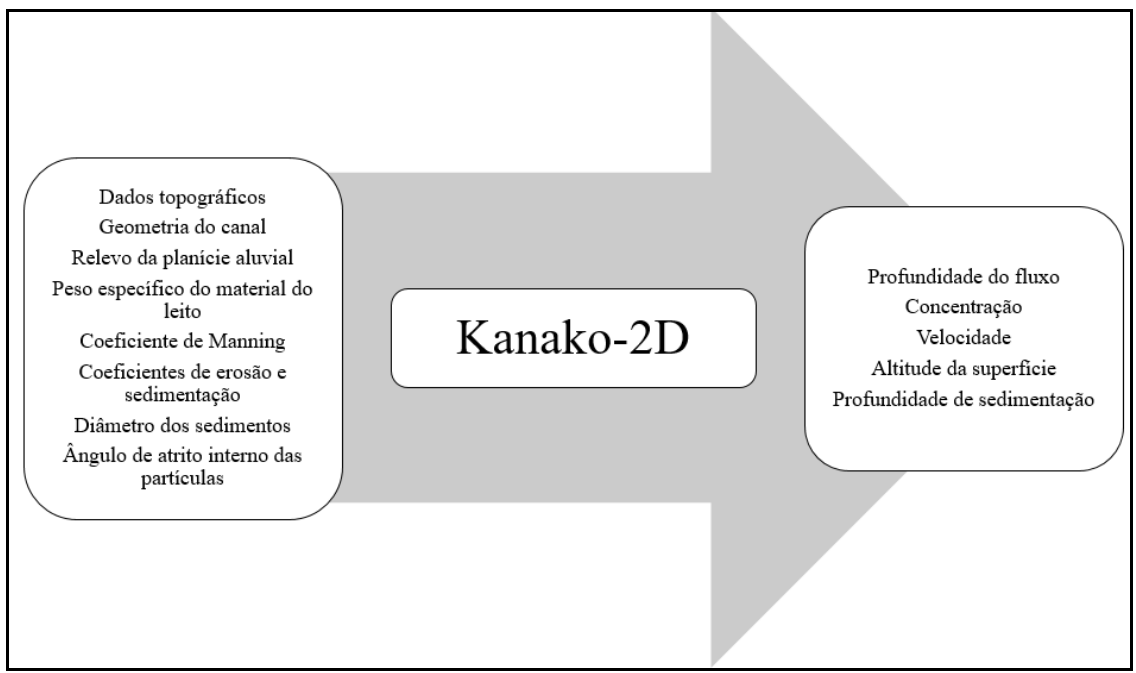

Figura 2 - Funcionamento simplificado do Kanako-2D

\section{Resultados e discusão}

Os resultados obtidos nas simulações são demonstrados na Figura 3. Após a obtenção de mapa de área de atingida, os valores do alcance, da área de erosão, da área de sedimentação e da área atingida foram calculados. Aqui nota-se que a área total atingida é somatório das áreas de erosão e de sedimentação.

A Figura 4 apresenta os resultados em termos de alcance, áreas de erosão, deposição e área total atingida pelo fluxo. Observa-se um decaimento potencial do tamanho dos sedimentos em função da distância percorrida pelo fluxo (Figura 4a). Para o diâmetro dos sedimentos igual a $0,15 \mathrm{~m}$, o fluxo percorreu uma distância de aproximadamente $1000 \mathrm{~m}$, enquanto que para o diâmetro de $3,0 \mathrm{~m}$, a distância percorrida foi de aproximadamente $300 \mathrm{~m}$. Quanto menor o tamanho dos sedimentos, maior foi o alcance do fluxo de detritos. Tal resultado coincide com a afirmação de Takahashi (2007) onde partículas menores apresentam maior mobilidade. 


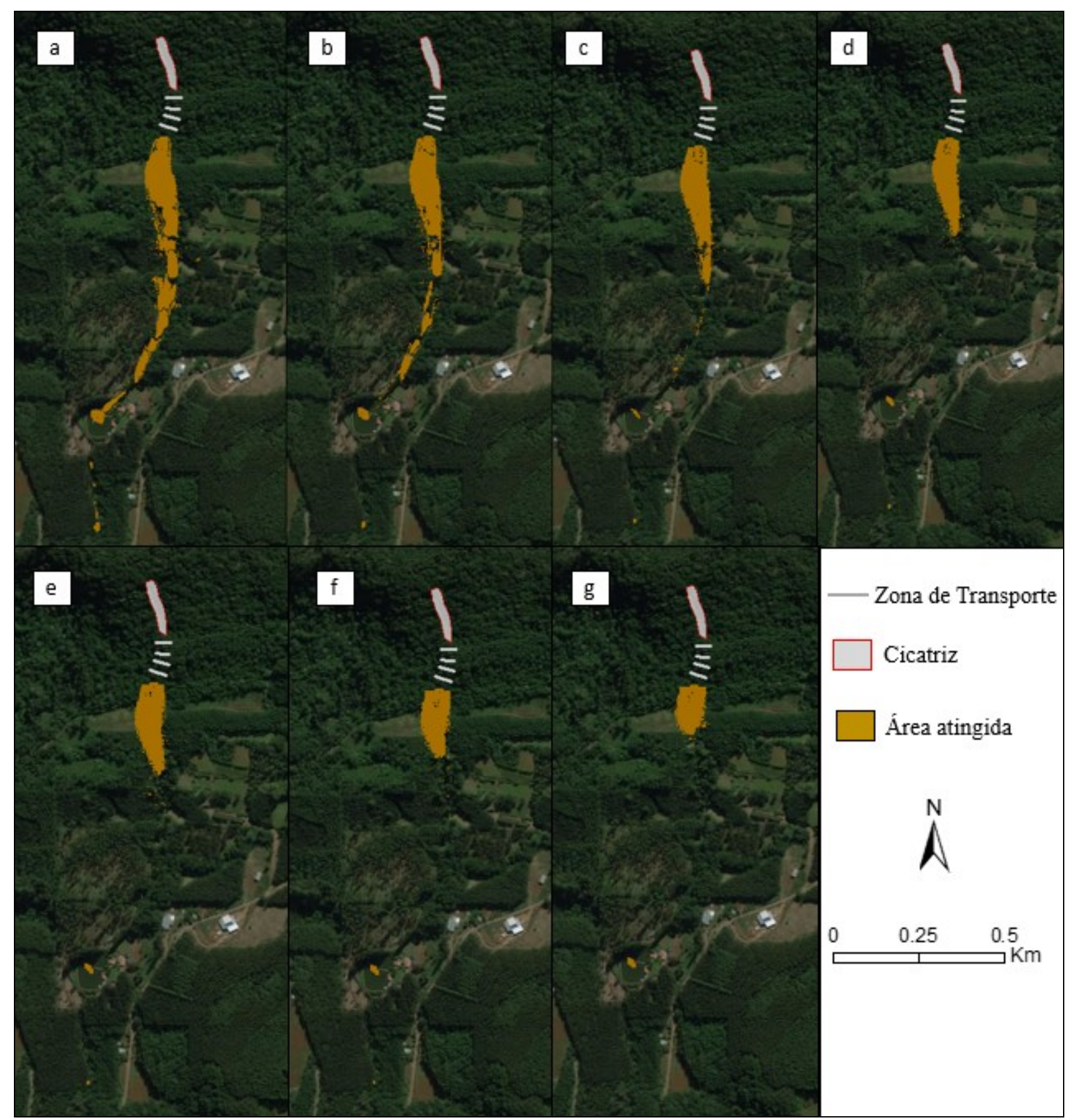

Figura 3 - Área atingida pelo fluxo de detritos: (a) $d=0,15 \mathrm{~m}$; (b) $d=0,30 \mathrm{~m}$; (c) $d=0,50 \mathrm{~m}$; (d) d=1,00 m; (e) d=1,30 m; (f) d=2,00 m; (g) d= $3,00 \mathrm{~m}$ 


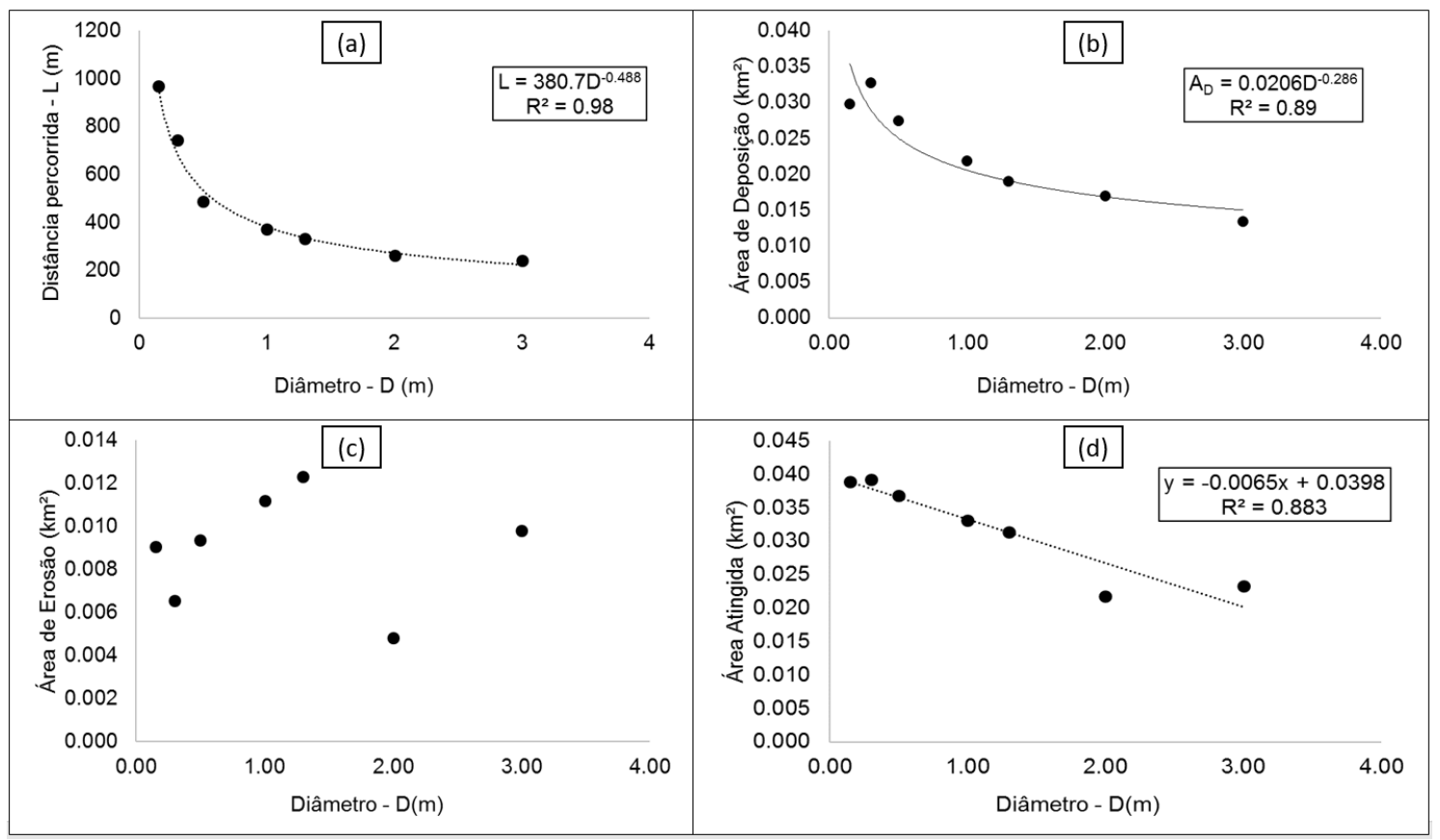

Figura 4 - Resultados com diferentes tamanhos de sedimentos: (a) distância de alcance; (b) área de deposição; (c) área de erosão; e (d) área atingida

Segundo o modelo de fluxo dilatante proposto por Takahashi (1991), a carga total das partículas afetadas pela flutuabilidade do fluido intersticial é sustentada pela força repulsiva produzida pela colisão das partículas. Isto sugere que partículas menores colidem mais ao longo da propagação do fluxo, permitindo maior alcance e maior área atingida (Figura 4d). A área atingida, considerada como a área afetada pelo fluxo, seja por deposição ou erosão de sedimentos, apresentou decaimento aproximadamente linear em relação ao tamanho dos sedimentos, sendo de 0,040 $\mathrm{km}^{2}$ para o tamanho de sedimentos de $0,15 \mathrm{~m}$ e de $0,025 \mathrm{~km}^{2}$ para sedimentos de $3,0 \mathrm{~m}$.

A área de deposição do material (Figura 4b) seguiu decaimento potencial com o tamanho dos sedimentos. Com sedimentos menores, a frente do fluxo de detritos pode ter inclinação menor que a inclinação do terreno, o que, segundo Takahashi (2007), pode facilitar a deposição de material quando o fluxo apresenta elevada fluidez. As áreas de deposição do material oscilaram valores entre $0,035 \mathrm{~km}^{2}$ e $0,015 \mathrm{~km}^{2}$ para os tamanhos de sedimentos de $0,15 \mathrm{~m}$ e $3,0 \mathrm{~m}$, respectivamente.

Quanto à área de erosão, não se encontraram relações claras com o tamanho dos sedimentos. Com o aumento do tamanho dos sedimentos o alcance foi diminuído, uma vez que é necessária maior energia para transportar o material, o que gerou aumento do espalhamento da área de erosão. Tal fato pode ser explicado com o aumento do poder destrutivo do material de movimento.

\section{Conclusões e recomentações}

O tamanho dos sedimentos fortemente influencia no alcance do fluxo de detritos, bem como na área total atingida e a área de deposição. Sedimentos menores apresentam maior mobilidade, permitindo que grandes distâncias sejam alcançadas e áreas maiores sejam atingidas.

A área de erosão dos sedimentos, no entanto, não está relacionada a uma tendência. Com o aumento do tamanho dos sedimentos o alcance foi reduzido e aumentou o espalhamento da área de erosão, o que pode estar 
relacionado ao aumento do poder destrutivo do material. No futuro, será necessária maior investigação acerca dos processos envolvidos na erosão causada por fluxos de detritos.

A utilização de simulação computacional através do modelo Kanako-2D permitiu maior conhecimento sobre os efeitos do tamanho dos sedimentos nos fluxos de detritos. Estudos sobre desastres relacionados a fluxo de detritos que utilizam modelagem computacional podem contribuir na previsão de cenários críticos e na identificação de áreas susceptíveis a estes fenômenos, sendo uma questão urgente para o Brasil.

\section{Referências}

AVELAR, A.S.; COELHO NETTO, A.L.; LACERDA, W.A.; BECKER, L.B.; MENDOÇA, M.B. (2011). Mechanisms of the recent catastrophic landslides in the mountainous range of Rio de Janeiro, Brazil. In: Forum, Proceedings of the Second World Landslide Rome, Italy, Oct 201, pp 265-270.

FLORES, C.A.; PÖTTER, R.O.; FASOLO, P.J.; HASENACK H. \& WEBER, E. Levantamento semidetalhado de solos: Região da Serra Gaúcha - Rio Grande do Sul. Porto Alegre: UFRGS/Embrapa Clima Temperado, 2007.

FRANK, B.; SEVEGNANI, L. (2009). Desastre de 2008 no Vale do Itajaí: Água, gente e política. Fundação Agencia de Água de Vale do Itajaí. Blumenau-SC, 192 p.

GODOY, J.V.Z.; BAUMBACH, M.F.; MICHEL, G.P.; ZAMBRANO, F.C.; BARRAGAN, M.L.M.; KOBIYAMA, M. Análise estatística de chuva na região da bacia do arroio Forromeco, RS, Brasil.. In: XXI Simpósio Brasileiro de Recursos Hídricos, 2015, Brasília. Segurança Hídrica e Desenvolvimento Sustentável. Porto Alegre: ABRH, 2015. p.1-8.

IRDR - Integrated Research on Disaster Risk. Peril Classification and Hazard Glossary. Beijing: Integrated Research on Disaster Risk, 2014. 24p. (IRDR DATA Publication No. 1).

JAKOB, M; HUNGR,O. (eds.) Debris-flow hazards and related phenomena. Berlin: Springer-Verlag, 2005. $739 \mathrm{p}$

KOBIYAMA, M.; MICHEL, G.P.; ENGSTER, E.C.; PAIXAO, M. A. Historical analyses of debris flow disaster occurrences and of their scientific investigation in Brazil. Labor \&Engenho, v.9, p.76-89, 2015.

MICHEL, G.P. Estimativa da profundidade do solo e seu efeito na modelagem de escorregamentos. 2015. 164 f. Tese (Doutorado)-Universidade Federal do Rio Grande do Sul, Instituto de Pesquisas Hidráulicas, Programa de Pós-Graduação em Recursos Hídricos e Saneamento Ambiental, Porto Alegre, BR-RS, 2015.

MINISTÉRIO DA INEGRAÇÃO NACIONAL Instrução normativa ～No. 01, de 24 de agosto de 2012. Brasília: Brasil, 2012. Diário Ocicial da União 169 e 170.

NAKATANI, K.; WADA, T.; SATOFUKA, Y.; MIZUYAMA, T.Development of “Kanako 2D (Ver.2.00)," a user-friendly one- and two-dimensional debris flow simulator equipped with a graphical user interface. International Journal of Erosion Control Engineering, v. 1, n. 2, p. 62-72, 2008.

REIS, J.T.; KOBIYAMA, M.; ZAMBRANO, F.C.; MICHEL, G.P. Correlação das variáveis para mapeamento de vulnerabilidade aos desastres hidrológicos no Arroio Forromeco-RS. Ciência e Natura, v.38, n.3, p.13611371, 2016.

RICKENMANN, D. Empirical Relationships for Debris Flows. Natural Hazards, v. 19, n. 1, p. 47-77, 1999.

TAKAHASHI, T. Progress in debris flow modeling. In: SASSA, K.; FUKUOKA, H.; WANG, F.; WABG, G. (eds.) Progress in landslide science. Heidelberg: Springer-Verlag, 2007. p.60-77. 
TAKAHASHI, T.; NAKAGAWA, H. Prediction of Stony Debris Flow Induced by Severe Rainfall. Sabo Gakkaishi, v. 44, n. 3, p. 12-19, 1991.

UCHIDA, T.; NISHIGUCHI, Y.; NAKATANI, K.; SATOFUKA, Y.; YAMAKOSHI, T.; OKAMOTO, A.; MIZUYAMA, T. . New numerical simulation procedure for large-scale debris flows (Kanako-LS). International Journal of Erosion Control Engineering, v. 6, n 2, p.58-67, 2013.

VIERO, A. C.; SILVA, D. R. A. (org.) Geodiversidade do estado do Rio Grande do Sul. Porto Alegre: CPRM, 2010. 